\title{
Antibiotic Treatment of Urinary Tract Infections (UTIs) In Primary Care: An Italian Pilot Study
}

Silvia Ussai ${ }^{1 *}$, Michele Rizzo ${ }^{2}$, Giovanni Liguori ${ }^{2}$, Paolo Umari ${ }^{2}$, Nicola Pavan ${ }^{2}$, Carlo Trombetta ${ }^{2}$, Tommaso Cai ${ }^{3}$ and Roberto Luzzati ${ }^{4}$

${ }^{1}$ Department of Infectious Disease, University Hospital of Udine, Italy

${ }^{2}$ Department of Urology, University of Trieste, Trieste, Italy

${ }^{3}$ Department of Urology, Santa Chiara Regional Hospital, Trento, Italy

${ }^{4}$ Department of Infectious Disease, University of Trieste, Italy

\begin{abstract}
Background: Urinary tract infections (UTIs) are extremely common. In the global scenario, due to the rise of antibiotic resistance worldwide, they present a substantial public health challenge. The aim of this cross-sectional study was to review antibiotic utilization in the management of UTIs in primary care practices in Italy.

Materials and methods: Patients in Italian primary care settings with a confirmed diagnosis of UTI and receiving antibiotic therapy were enrolled in a cloud-based pharmacovigilance study.

Results: The study included 5232 patients (3903 females, 1329 males) who had been prescribed antibiotics for UTI between July 2014 and December 2015. Quinolones were prescribed for 4889 patients (94\%) as the first line treatment. In the 14-days follow-up period, 3181 patients $(60 \%)$ received at least one different antibiotic medication. Fifty-eight percent $(n=1844)$ of patients further received antibiotics on day 2 and day 3 of the follow-up period. Different quinolones and cephalosporins were the most prescribed medications in this sub-population.

Conclusion: The study raises concerns about antibiotic and specifically quinolone overuse in Italian UTI outpatients. Policy-making bodies and professional societies should prioritize reducing the inappropriate use of antibiotics. This can be achieved through antimicrobial stewardship programs, which are one of the primary solutions to address the growing problem of antimicrobial drugs resistance.
\end{abstract}

Keywords: UTI; Antibiotic; Resistance; Quinolone

\section{Introduction}

Urinary tract infections (UTIs) have become the most frequent bacterial infections worldwide. It is estimated that UTIs contribute to seven million hospital visits, one million emergency consultations and more than 100.000 hospitalizations [1] annually in the U.S alone.

Primary care settings often diagnose and manage UTIs [2]. They often empirically manage a broad spectrum of pathologies from acute cystitis to pyelonephritis [3].

In addition, they care for a broad range of patients, ranging from low risk groups such as young adults to vulnerable populations such as elderly patients, pregnant women [4], spinal cord injury patients [5], and patients requiring permanent or intermittent bladder catheterization [6].

When deciding on empiric therapy for a UTI, local resistance patterns to antibiotics are an important factor in choice of therapy [7]. Given this consideration, antibiotic choice should be based not only on efficacy and safety [8], but also, on the concept that broadspectrum antibiotics should be spared in order to safeguard their future effectiveness [9].

Primary care providers are critical to appropriately using antibiotics to manage UTIs, as they manage the majority of cases [10]. Scientific societies propose guidelines and recommendations for UTI management, which are periodically reviewed. Despite of this, common clinical practices appear poorly adherents to the best practice recommendations. Audit control procedures are necessary to identify critical issues, sub-optimal behaviour and provide solution to problems.

\section{Aim}

The goal of this study was to perform an analysis of the antibiotics Italian UTI patients receive in primary care. Authors also assessed if changes were made to the empiric therapy within the first 14 days of treatment.

\section{Materials and Methods}

All the patients included in this analysis lived in the northeast of Italy. During the study period, the study population received a primary care evaluation, a diagnosis of UTI, and the prescription of at least one antibiotic drug.

\section{Inclusion criteria}

a. Patients in primary care setting receiving an antibiotic prescription during the study period (18 months) and confirmed for UTI diagnosis;

b. Patients aged 14 years and over;

c. Patients agreeing to participate in the program.

*Corresponding author: Silvia Ussai, Department of Infectious Disease, University Hospital of Udine, Piazzale della Misericordia 15, 33100, Udine, Italy, Tel: 0432559111; E-mail: ussai.silvia@gmail.com

Received: June 18, 2016; Accepted July 06, 2016; Published July 11, 2016

Citation: Ussai S, Rizzo M, Liguori G, Umari P, Pavan N, et al. (2016) Antibiotic Treatment of Urinary Tract Infections (UTIs) In Primary Care: An Italian Pilot Study. J Pharmacovigilance 4: 215. doi: 10.4172/2329-6887.1000215

Copyright: ( 2016 Ussai S, et al. This is an open-access article distributed under the terms of the Creative Commons Attribution License, which permits unrestricted use, distribution, and reproduction in any medium, provided the original author and source are credited. 


\section{Therapy assessment}

Electronic health records (EHR) of patients were obtained through a cloud-based service using a closed loop pharmacovigilance system that recorded and updated all antibiotics taken during treatment.

The EHR was designed to identify and maintain a single patient record for each subject, report patient demographic information, and to create and maintain patient-specific medications lists.

This EHR has security features allowing communication amongst all members of the health care team and the researchers.

The diagnosis of UTI was confirmed by reviewing patient charts. The authors also identified new antibiotic prescriptions within the first 14 days of diagnosis, specifically on days 2, 3, 7 and 14 of the followup period. Statistical analysis was performed using $\mathrm{SAS}^{\odot}$ statistical package 9.3 (SAS Institute Inc., Cary, NC, USA).

\section{Results}

The study included 5232 patients (3903 females, 1329 males) who had been prescribed antibiotics for UTI management between July 2014 and December 2015. Graph 1 describes the age and gender distribution among the cohort.

Within the study, almost all infections were seen in adults over age $19,99.4 \%$. Women in the age group between 20 and 79 years of age had $76.9 \%$ of all infections.

The highest incidence of UTI in females was found in the age group between 30 and 59 years as more than $50 \%$ of the UTI cases were detected in this age group. In this study, $94 \%$ of the patients $(n=4889)$ received a quinolone as the first line treatment for UTI (Graph 2). About 3\% $(n=169)$ received Cephalosporins and 2\% received Betalactams.

The other antibacterial drugs prescribed to patients included fosfomycin, macrolides, lincosamides and sterptogramines antibiotics. A total of 3181 patients (60\%) further received one or more new antibiotics in the 14 days follow-up period (Graph 3).

About $67 \%$ of the patients $(n=2135)$ further received 1 additional antibiotic for UTI treatment. A total of $588(18 \%)$ patients were prescribed 2 new medications and 399 patients (12.5\%) received 3 to 6 additional drugs since the first prescription (Table 1).

In this study, the 4 classes of antimicrobial drugs prescribed after empiric therapies were: beta-lactams, tetracycline, other quinolones, and cephalosporins. At days 2 and 3, about 58\% of the patients $(n=1844)$ had received new antibiotics; the new medications were primarily beta-lactams and cephalosporin (Graph 4). On day 7 a spike in the prescribing of alternative quinolones was seen. By the 14th day of the follow-up period, $23 \%(n=733)$ of the study cohort had received new antibiotic prescription, often tetracycline (Graph 5).

\section{Discussion}

This is the first Italian cross-sectional pilot study performed in the primary care arena to study antibiotic management in UTI patients. The study population usually received quinolones $(n=4889)$, and, to a lesser extent, cephalosporin and beta-lactams $(n=345)$ as the first line of treatment. In the 14 days following the first prescription, 3181 patients were prescribed a different antibiotic. It was found that by day 2 and 3 of the follow-up, about $58 \%$ of patients had already started on

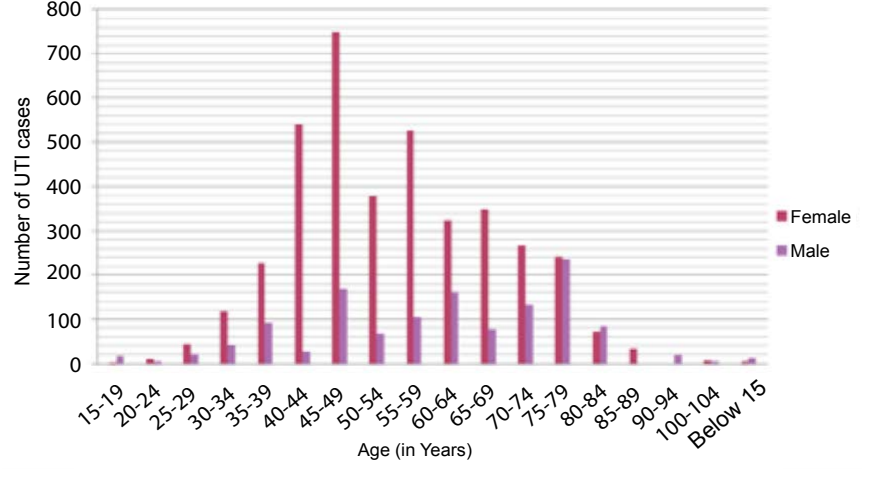

Graph 1: Age and gender distribution, UTI cohort

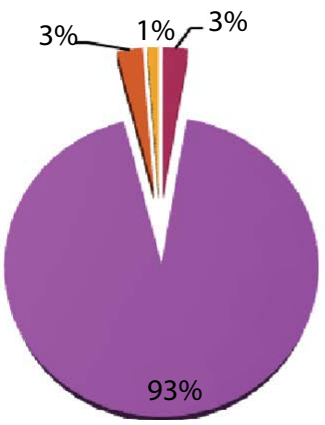

- Beta-lactams

Quinolones

- Other beta-lactams

- Other antibacterials

Graph 2: Prevalence of use of antibiotics for UTI treatment

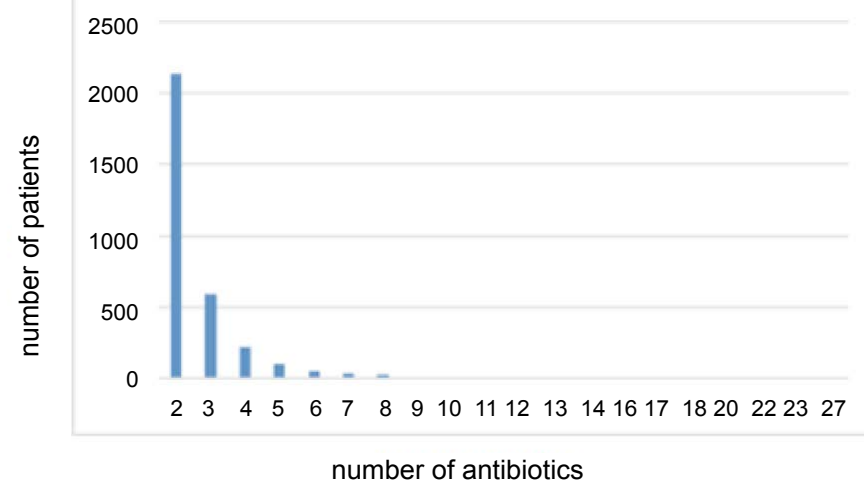

Graph 3: Number of patients Vs Number of new antibiotic prescription for UTI

Patients receiving new antibiotics during the follow-up

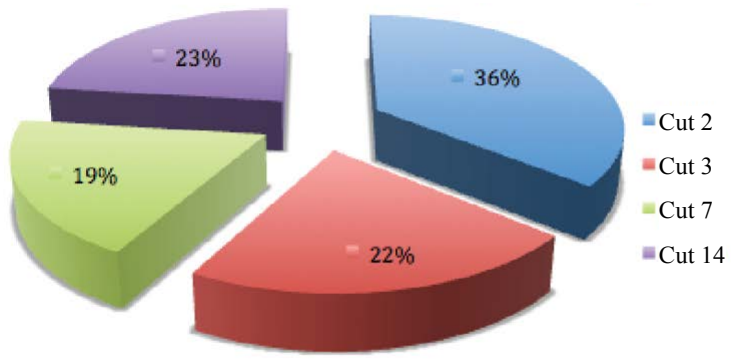

Graph 4: Percentage of patients receiving new antibiotics during the follow-up period 


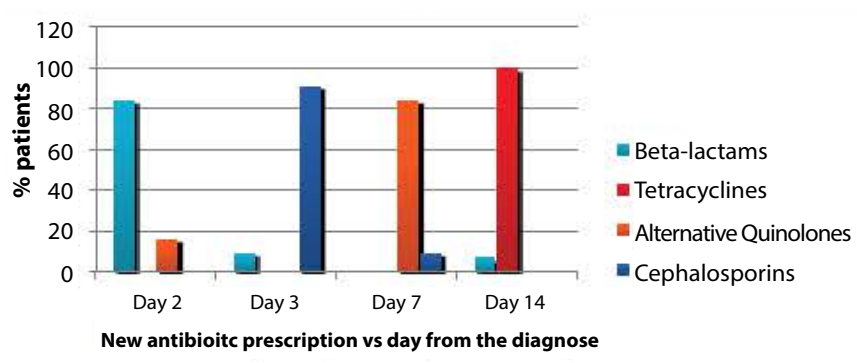

Graph 5: New antibiotic class distribution vs Follow-up days

\begin{tabular}{|c|c|}
\hline No. of antibiotics & No. of patients \\
\hline 1 & 2135 \\
\hline 2 & 588 \\
\hline 3 & 219 \\
\hline 4 & 98 \\
\hline 5 & 47 \\
\hline 6 & 35 \\
\hline 7 & 21 \\
\hline 8 & 8 \\
\hline 9 & 7 \\
\hline 10 & 4 \\
\hline 11 & 3 \\
\hline 12 & 7 \\
\hline 13 & 1 \\
\hline 14 & 1 \\
\hline 15 & 1 \\
\hline 16 & 2 \\
\hline 19 & 1 \\
\hline 21 & 1 \\
\hline 22 & 1 \\
\hline 24 & 1 \\
\hline Total & 3181 \\
\hline
\end{tabular}

Table 1: Number of patients receiving additional antibiotics.

new antibiotic. The study lacked adequate data about the clinical and microbiological details of the patient's diagnosis, however, as most of the patients were adult females it was clinically concluded that for most patients the uncomplicated UTIs was most likely cystitis.

According to the European Urology Guidelines for UTIs, neither quinolones nor cephalosporins are appropriate for the empiric treatment of uncomplicated cystitis [11]. Clearly our work shows that these guidelines were generally not followed.

The study did not have sufficient access to patient data to determine why these changes were made; however, we believe urine culture results, persistent symptoms or worsening of symptoms all could explain the changes in therapy.

The new antibiotic prescriptions may have been based on the incomplete remission of the patients' symptoms or due to the occurrence of drug related adverse events. Andrew Cole, in a recent survey in United Kingdom involving 1000 General Physicians (GP), found that $55 \%$ of the GPs unwillingly prescribed antibiotics under the pressure of patients or patients, even when antibiotic therapy is not considered necessary [12]. Potential antibiotic overuse raises concern about resistance to a particular UTI drug or class of antibiotics. This study reveals the need for robust drug utilization review program and guidelines in the primary care management of UTI.

\section{Limitation}

This study has a few clear limitations. Foremost, the study lacks adequate clinical and microbiological data to determine why antibiotics were changed or if adverse events occurred. Secondly, therapy was evaluated retrospectively based on patient's files and some data may not have been recorded.

\section{Conclusion}

Our study clearly demonstrates that Italian primary-care physicians are not following best practices when empirically managing UTI's and often change their prescribing patterns during therapy. Policymaking bodies and professional societies should prioritize, reducing the inappropriate use of antibiotics through antimicrobial stewardship programs, which is one of the primary solutions to address the growing problem of antimicrobial resistance.

\section{References}

1. Foxman B (2002) Epidemiology of urinary tract infections: incidence, morbidity, and economic costs. Am J Med 113 Suppl 1A: 5S-13S

2. Little P, Turner S, Rumsby K, Warner G, Moore M, et al. (2006) Developing clinical rules to predict urinary tract infection in primary care settings: sensitivity and specificity of near patient tests (dipsticks) and clinical scores. $\mathrm{Br} \mathrm{J}$ Gen Pract 56: 606-612.

3. Fair RJ, Tor $Y(2014)$ Antibiotics and bacterial resistance in the $21^{\text {st }}$ century. Perspect Medicin Chem 6: 25-64.

4. Schieve LA, Handler A, Hershow R, Persky V, Davis F (1994) Urinary tract infection during pregnancy: its association with maternal morbidity and perinatal outcome. Am J Public Health 84: 405-410.

5. Esclarín De Ruz A, García Leoni E, Herruzo Cabrera R (2000) Epidemiology and risk factors for urinary tract infection in patients with spinal cord injury. J Urol 164: 1285-1289.

6. Hooton TM, Stam WE (1991) Management of acute uncomplicated urinary tract infection in adults. Med Clin North Am 75: 339-357.

7. Russell $G$ (2016) Antibiotic resistance in children with $E$ coli urinary tract infection. BMJ 352: i1399.

8. Lee CR, Cho IH, Jeong BC, Lee SH (2013) Strategies to minimize antibiotic resistance. Int J Environ Res Public Health 10: 4274-4305.

9. Hay AD (2010) Managing UTI in primary care: should we be sending midstream urine samples? Br J Gen Pract 60: 479-480.

10. Naber KG, Bergman B, Bishop MC, Bjerklund-Johansen TE, Botto $H$, et al (2001) EAU guidelines for the management of urinary and male genital tract infections. Urinary Tract Infection (UTI) Working Group of the Health Care Office (HCO) of the European Association of Urology (EAU). Eur Urol 40: 576588

11. Kunin CM, McCormack RC (1966) Prevention of catheter-induced urinary-tract infections by sterile closed drainage. N Engl J Med 274: 1155-1161.

12. Cole A (2014) GPs feel pressurised to prescribe unnecessary antibiotics, survey finds. BMJ 349: g5238. 